\title{
COOPERATIVE CONTROL OF MULTIPLE SPACE MANIPULATORS
}

\author{
Gary E. Yale* \\ Phillips Laboratory \\ Kirtland AFB, NM 87117
}

\author{
Brij N. Agrawal ${ }^{\dagger}$ \\ Naval Postgraduate School \\ Monterey, CA 93940, USA
}

\begin{abstract}
This paper concerns the cooperative control of multiple manipulators attached to the same base as they reposition a common payload. The theory is easily applied to inertially based problems as well as space based free-floating platforms. The system equations of motion are developed as well as a Lyapunov based controller which ensures stability. The closed chain aspect of the problem reduces the system's degrees of freedom resulting in more actuators than degrees of freedom. This actuator redundancy is used to minimize a weighted norm of the actuator torques. A polynomial reference trajectory describes the path the payload will follow. The disturbance torque transmitted to the spacecraft centerbody by the motion of the manipulators is minimized by altering the order of the reference trajectory polynomial and its coefficients. Results from a two dimensional, dual arm configuration are included. Compared to the Lyapunov point controller alone, a fifth order polynomial reference trajectory leads to superior performance in terms of actuator torque magnitudes, spacecraft centerbody attitude control, and payload repositioning accuracy and time. An eighth order polynomial reference trajectory results in only minimal improvement over the fifth order case. A modified Lyapunov controller which approximates a PD controller produces results better than the Lyapunov point controller but not as good as either reference trajectory simulation.
\end{abstract}

\section{Background}

Space based robotics platforms experience conditions unlike those of their terrestrial counterparts. With respect to the dynamics of the systems, the most notable difference is the absence of a fixed base on which to locate the manipulators. The consequence of this difference is that motion of the space based manipulator transmits forces and moments to its mounting base resulting in translation and rotation of the base itself. ${ }^{1-2}$ Generally, this motion is unwanted because the attitude control subsystem of the vehicle must

Copyright ( $\odot 1994$ by Yale and Agrawal. Published by American Institute of Aeronautics and Astronautics, Inc. with permission.

\footnotetext{
* Major, United States Air Force

${ }^{\dagger}$ Professor, Department of Aeronautics and Astronautics
}

compensate. One can find the spacecraft attitude disturbance caused by manipulator motion and use that information to command reaction wheels on the main body. ${ }^{3}$ Better yet, one should try to minimize the attitude disturbance the manipulators transmit to the main body. For a spacecraft with a single manipulator with redundant kinematics, the excess degrees of freedom can be used to minimize reactions transmitted to the main body. ${ }^{4}$ If the manipulator is sufficiently redundant, the attitude disturbances may be eliminated altogether.' For spacecraft with multiple manipulators, cooperative control takes on more than one meaning. In one case, one manipulator repositions an object while a second manipulator, which is not grasping the object, moves to provide counterbalancing torques on the main body thereby reducing the spacecraft attitude disturbance. ${ }^{6}$ A more traditional concept of cooperative control of multiple manipulators assumes the manipulators are each in contact with the payload. One control strategy developed for a fixed base system controls the payload position and its internal forces using a Lyapunov controller or an adaptive controller. ${ }^{7}$ A space based version uses object impedance control to position the payload and control its internal forces. ${ }^{8}$ In this paper, cooperative control means multiple manipulators grasping a common object moving in harmony to reposition the object. When more than one manipulator grasps an object, the actuator redundancy created by the closed chain dynamics permits tradeoffs to be made regarding how the actuators are used. The user has great flexibility in choosing to what degree each actuator is involved in repositioning the payload.

The following development of an analytical model is based on a multiple-manipulator space robotics system. The manipulators already have a firm grasp of the payload. The initial conditions for the system are known although there may be some error in these values. Desired final conditions are also known. The equations of motion are derived from Lagrange's equations. This results in a set of second order, nonlinear, coupled, differential equations. Trajectory control connects the boundary conditions. With reference trajectories, actuator inputs are computed by means of inverse kinematics. The actuator inputs are modified using a Lyapunov derived controller. The controller compares the reference trajectories with the actual trajectories. The reference trajectories are selected by means of an optimization 
algorithm to reduce the attitude disturbance on the main spacecraft.

\section{Equations of Motion}

Development of the analytical model is predicated on establishing the variables and coordinates systems which will describe the system. The most general case is for a spacecraft with $\mathrm{n}$ manipulators involved in controlling the positioning of a common payload. The centerbody, manipulator links, and payload are rigid bodies. A semi-inertial axis system is located somewhere on the centerbody. The origin of this coordinate system remains fixed to the spacecraft. However, this coordinate frame maintains an inertial orientation. The centerbody attitude is referenced to this coordinate frame. Each manipulator link has its own set of body axes. The axes for each link are attached at the point of rotation nearest the centerbody. The $\mathrm{x}$ axis points along the longitudinal axis of the link. The angles which describe link orientation are joint angles with two subscripts. The first subscript indicates which manipulator the link belongs to. The second subscript indicates the particular link of that manipulator. The links are numbered outward from the centerbody. The payload orientation is referenced back to the coordinate frame on the centerbody. The dual two-link manipulator case is shown in Figure 1. To eliminate gravity,

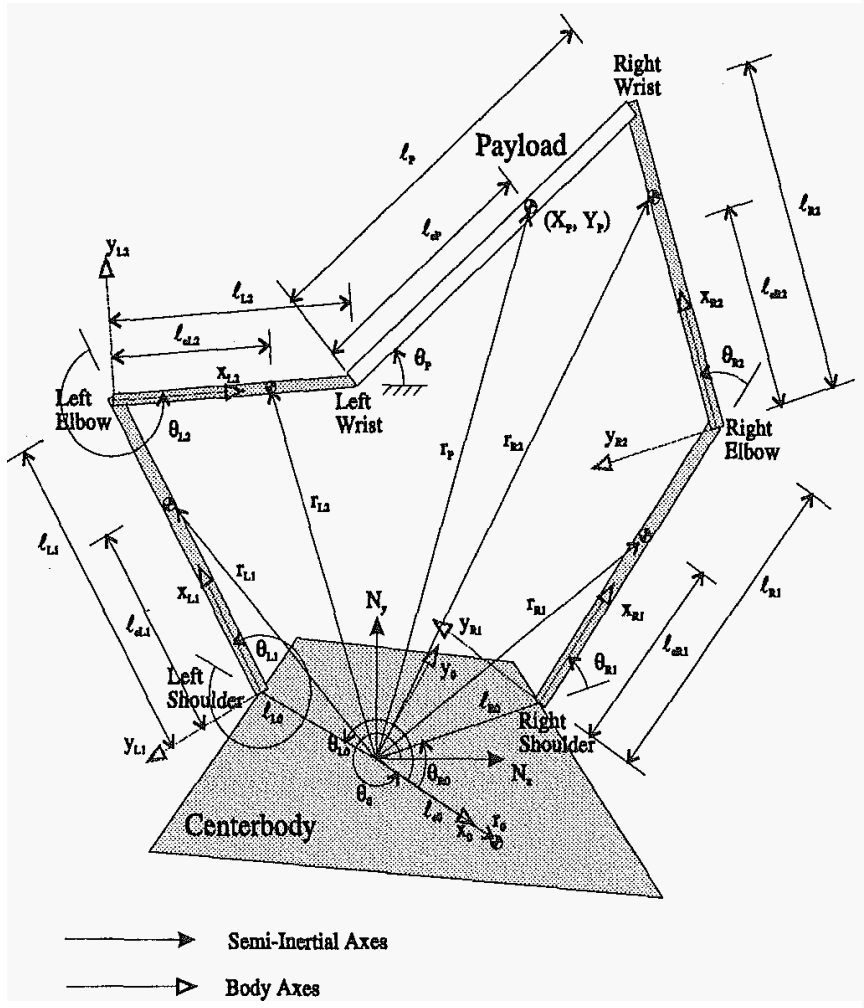

this two dimensional model is in the horizontal plane. The $\mathrm{z}$ axis is perpendicular to the plane of the motion. The generalized coordinates are

$$
q=\left[\begin{array}{llllllll}
\theta_{0} & \theta_{L 1} & \theta_{L 2} & \theta_{R 1} & \theta_{R 2} & \theta_{P} & X_{P} & Y_{P}
\end{array}\right]^{T}
$$

They include centerbody attitude, left and right arm joint angles, payload attitude, and payload center of mass coordinates. Like the centerbody angle, the payload angle is referenced to an inertial coordinate frame. The mounting location for the left and right shoulders are given by the two constant angles $\theta_{L 0}$ and $\theta_{R 0}$. The distances from the semiinertial coordinate frame to the shoulders are $l_{L 0}$ and $l_{R 0}$. Link lengths are designated as $l_{i}$. Distances to link centers of mass contain the letter $\mathrm{c}$ as a subscript. The control actuators for this system consist of a reaction wheel mounted on the centerbody and joint motors at the shoulder, elbow, and wrist of each manipulator. The resulting control input vector is

$$
u=\left[u_{w h} u_{L S} u_{L E} u_{L W} u_{R S} u_{R E} u_{R W}\right]^{\mathrm{T}}
$$

The first element is the reaction wheel torque. The next three elements are the shoulder, elbow and wrist torques for the left manipulator. The final three elements are for the right manipulator.

The equations of motion for this system are developed using Lagrange's equations for a dynamic system with holonomic constraints (Eq. (3)). The constraints are due to the geometry of the system.

$$
\frac{d}{d t}\left(\frac{\partial L}{\partial \dot{q}}\right)-\frac{\partial L}{\partial \underline{q}}=\underline{Q}+A^{T} \underline{\lambda}
$$

subject to the constraint equations $\boldsymbol{A q}+A_{0}=0$

$$
\mathrm{L}=\mathrm{T} \cdot \mathrm{V}
$$

$\mathrm{T}$ is kinetic energy

$\mathrm{V}$ is potential energy

$q$ are the generalized coordinates

$\dot{q}$ are the generalized velocities

$\boldsymbol{Q}$ are the applied nonconservative forces

$A^{\gamma} \lambda$ are the constraint forces

Because of the closed chain nature of the system, the choice of generalized coordinates in Eq. (1) is not a minimum coordinate formulation. Consequently, the constraint forces (the last term in Eq. (3)) will be nonzero.

Beginning with Lagrange's equation, the equations of motion can be rearranged into the alternate form

$$
M(\underline{q}) \underline{q}+G(\underline{q}, \dot{q})+\frac{\partial V}{\partial \underline{q}}=\underline{Q}+A^{T} \underline{\lambda}
$$

FIGURE 1. Dual Two-Link Manipulator Case 
$\mathrm{M}$ is the inertia matrix. It is a function of the generalized coordinates and can be found by expressing the kinetic energy in the form of Eq. (5).

$$
T=\frac{1}{2} \dot{q}^{T}[M(\underline{q})] \underline{\dot{q}}
$$

For the dual two-link manipulator case shown in Figure 1, the inertia matrix is given by

$$
\begin{aligned}
& M=\left[\begin{array}{cccccccc}
M_{11} & M_{12} & M_{13} & M_{14} & M_{15} & 0 & 0 & 0 \\
M_{21} & M_{22} & M_{23} & 0 & 0 & 0 & 0 & 0 \\
M_{,} & M_{32} & M_{33} & 0 & 0 & 0 & 0 & 0 \\
M_{41} & 0 & 0 & M_{44} & M_{45} & 0 & 0 & 0 \\
M_{51} & 0 & 0 & M_{,}, & M_{55} & 0 & 0 & 0 \\
0 & 0 & 0 & 0 & 0 & I_{P} & 0 & 0 \\
0 & 0 & 0 & 0 & 0 & 0 & m_{P} & 0 \\
0 & 0 & 0 & 0 & 0 & 0 & 0 & \mathrm{~m}_{P}
\end{array} \mid\right. \\
& M_{55}=I_{R 2}+m_{R 2} l c_{R 2}^{2} \\
& M_{45}=M, \quad=M_{55}+m_{R 2} l_{R 1} l c_{R 2} \cos \theta_{R 2} \\
& =M_{51}=M_{45}+m_{R 2} l_{R 0} l c_{R 2} \cos \left(\theta_{R 1}\right. \\
& M_{44}=M_{45}+I_{R 1}+m_{R 2} l_{R 1} l c_{R 2} \cos \theta_{R 2}+m_{R 1} l c_{R 1}^{2}+m_{R 2} l_{R 1}^{2}(10) \\
& M_{14}=M_{41}=M_{44}+l_{R 0}\left(m_{R 1} l c_{R 1}+m_{R 2} l_{R 1}\right) \cos \theta_{R 1} \\
& +m_{R 2} l_{R 0} l c_{R 2} \cos \left(\theta_{R 1}+\theta_{R 2}\right) \\
& M_{33}=I_{L 2}+m_{L 2} l c_{L 2}^{2} \\
& M_{23}=M_{32}=M_{33}+m_{L 2} l_{L 1} l c_{L 2} \cos \theta_{L 2} \\
& M_{13}=M_{31}=M_{23}+m_{L 2} l_{L 0} l c_{L 2} \cos \left(\theta_{L 1}+\theta_{L 2}\right) \\
& M_{22}=M_{23}+I_{L 1}+m_{L 2} l_{L 1} l c_{L 2} \cos \theta_{L 2}+m_{L 1} l c_{L 1}^{2}+m_{L 2} l_{L 1}^{2} \\
& M_{12}=M_{21}=M_{22}+l_{L 0}\left(m_{L 1} l c_{L 1}+m_{L 2} l_{L 1}\right) \cos \theta_{L 1} \\
& +m_{L 2} l_{L 0} l c_{L 2} \cos \left(\theta_{L 1}+\theta_{L 2}\right) \\
& M_{11}=I_{0}+m_{0} l c_{0}^{2}+2 m_{R 2} l_{R 0} l c_{R 2} \cos \left(\theta_{R 1}+\theta_{R 2}\right) \\
& +\left(m_{R 1}+m_{R 2}\right) l_{R 0}^{2}+2 l_{R 0}\left(m_{R 1} l c_{R 1}+m_{R 2} l_{R 1}\right) \cos \theta_{R 1} \\
& +\left(m_{L 1}+m_{L 2}\right) l_{L 0}^{2}+2 l_{L 0}\left(m_{L 1} l c_{L 1}+m_{L 2} l_{L 1}\right) \cos \theta_{L 1} \\
& +2 m_{L 2} l_{L 0} l c_{L 2} \cos \left(\theta_{L 1}+\theta_{L 2}\right)+M_{22}+M_{44}
\end{aligned}
$$

Because the generalized coordinate for the payload attitude is referenced to an inertial coordinate frame, the inertia matrix is decoupled between the payload and the rest of the system. Coupling does exist between the spacecraft centerbody and each of the manipulators.
The $\mathrm{G}$ matrix contains all of the centripetal and Coriolis terms. It is most easily found using the following equations

$$
\begin{gathered}
G(\underline{q}, \underline{\dot{q}})=\left[\begin{array}{cc}
\dot{q}^{T} C^{(1)} & \dot{q} \\
\dot{q}^{T} C^{(2)} & \dot{q} \\
\dot{q}^{T} C^{(8)} & \dot{q}
\end{array}\right] \\
C_{j k}^{(i)}=\frac{1}{2}\left(\frac{\partial M_{i j}}{\partial q_{k}}+\frac{\partial M_{i k}}{\partial q_{j}}-\frac{\partial M_{j k}}{\partial q_{i}}\right)
\end{gathered}
$$

where $C_{j k}^{(i)}$ is the $\mathrm{jk}^{\text {th }}$ element in the $\mathrm{i}^{\text {th }} \mathrm{C}$ matrix.

The nonconservative forces, Q, may be expressed as the product of a control influence matrix and the input vector ( $Q=\boldsymbol{B} \boldsymbol{u})$. For the configuration of Figure 1, the control influence matrix is

$$
B=\left[\begin{array}{ccccccc}
1 & 0 & 0 & -1 & 0 & 0 & -1 \\
0 & 1 & 0 & -1 & 0 & 0 & 0 \\
0 & 0 & 1 & -1 & 0 & 0 & 0 \\
0 & 0 & 0 & 0 & 1 & 0 & -1 \\
0 & 0 & 0 & 0 & 0 & 1 & -1 \\
0 & 0 & 0 & 1 & 0 & 0 & 1 \\
0 & 0 & 0 & 0 & 0 & 0 & 0 \\
6 & 0 & 0 & 0 & 0 & 0 & 0
\end{array}\right]
$$

The constraints matrix, $\mathbf{A}$, is derived by writing the system constraints in the Pfaffian form as

$$
A \underline{q}+A_{0}=0
$$

The system constraints are those equations which describe the closed chain geometry of the system. For example, the dual two-link manipulator system is eighth order. Because this system has only four degrees of freedom, an additional four equations are needed to describe the constraints. These equations come from geometric relationships describing the payload center of mass Cartesian coordinates in terms of the left and right arm generalized coordinates.

$$
\begin{aligned}
X_{P}= & l_{L 0} \cos \left(\theta_{0}+\theta_{L 0}\right)+l_{L 1} \cos \left(\theta_{0}+\theta_{L 0}+\theta_{L 1}\right) \\
& +l_{L 2} \cos \left(\theta_{0}+\theta_{L 0}+\theta_{L 1}+\theta_{L 2}\right)+l c_{P} \cos \theta_{P} \\
Y_{P}= & l_{L 0} \sin \left(\theta_{0}+\theta_{L 0}\right)+l_{L 1} \sin \left(\theta_{0}+\theta_{L 0}+\theta_{L 1}\right) \\
& +l_{L 2} \sin \left(\theta_{0}+\theta_{L 0}+\theta_{L 1}+\theta_{L 2}\right)+l c_{P} \sin \theta_{P} \\
X_{P}= & l_{R 0} \cos \left(\theta_{0}-\theta_{R 0}\right)-l_{R 1} \cos \left(\theta_{0}+\theta_{R 0}-\theta_{R 1}\right) \\
& +l_{R 2} \cos \left(e,+\theta_{R 0}+\theta_{R 1}+\theta_{R 2}\right)-\left(l_{P}-l c_{P}\right) \cos \theta_{P}
\end{aligned}
$$




$$
\begin{aligned}
Y_{P}= & l_{R 0} \sin \left(\theta_{0}+\theta_{R 0}\right)+l_{R 1} \sin \left(\mathbf{0},+\theta_{R 0}+\theta_{R 1}\right) \\
& +l_{R 2} \sin \left(\theta_{0}+\theta_{R 0}+\theta_{R 1}+\theta_{R 2}\right)-\left(l_{P}-l c_{P}\right) \sin \theta_{P}
\end{aligned}
$$

To get the Pfaffian form of Eq. (21), differentiate Eqs. (22-25) and rearrange terms. The following equations express the result. The constant term, $\mathrm{A}_{0}$, is a zero vector.

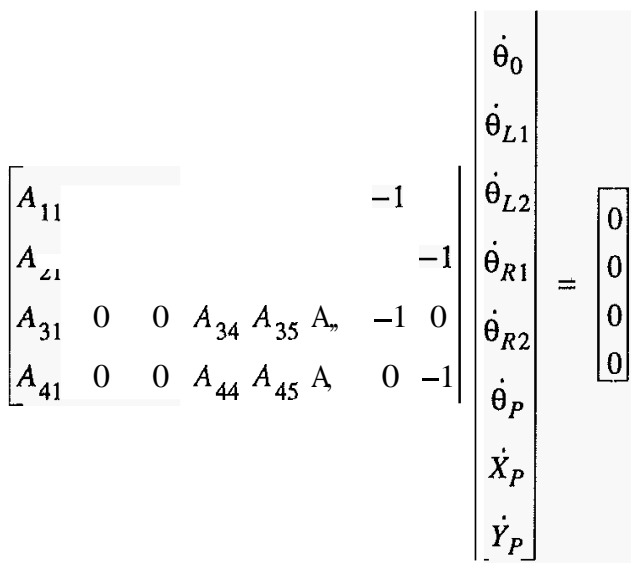

$$
\begin{aligned}
& \mathrm{A}, \quad=-l c_{P} \sin \theta_{P} \\
& \mathrm{~A}_{n}=l c_{P} \cos \theta_{P} \\
& \mathrm{~A}_{,},=\left(l_{P}-l_{c_{P}}\right) \sin \theta_{P} \\
& \mathrm{~A},=-\left(l_{P}-l c_{P}\right) \cos \theta_{P} \\
& A_{45}=l_{R 2} \cos \left(\theta_{0}+\theta_{R 0}+\theta_{R 1}+\theta_{R 2}\right) \\
& \mathrm{A},=A_{45}+I_{n}, \quad \cos \left(\mathbf{0},+\theta_{R 0}+\theta_{R 1}\right) \\
& A_{41}=\mathrm{A} 44+l_{R 0} \cos \left(\theta_{0}+\theta_{R 0}\right) \\
& \mathrm{A}_{,}=-l_{R 2} \sin \left(\theta_{0}+\theta_{R 0}+\theta_{R 1}+\theta_{R 2}\right) \\
& A_{34}=\mathrm{A}_{,}, \mathrm{I}, \quad \sin \left(\mathbf{0},+\theta_{R 0}+\theta_{R 1}\right) \\
& A_{31}=A_{34}-l_{R 0} \sin \left(0,+\theta_{R 0}\right) \\
& A_{23}=l_{L 2} \cos \left(\theta_{0}+\theta_{L 0}+\theta_{L 1}+\theta_{L 2}\right) \\
& A_{22}=A_{23}+l_{L 1} \cos \left(\theta_{0}+\theta_{L 0}+\theta_{L 1}\right) \\
& \mathrm{A},=A_{22}+l_{L 0} \cos \left(\theta_{0}+\theta_{L 0}\right) \\
& \mathrm{A}_{,}=-l_{L 2} \sin \left(\theta_{0}+\theta_{L 0}+\theta_{L 1}+\theta_{L 2}\right) \\
& \mathrm{A}, \quad=A_{13}-l_{L 1} \sin \left(\theta_{0}+\theta_{L 0}+\theta_{L 1}\right) \\
& \mathrm{A},,=A_{12}-l_{L 0} \sin \left(\theta_{0}+\theta_{L 0}\right)
\end{aligned}
$$

\section{Integrating the Equations of Motion}

After substituting the matrix form of the generalized forces into the equations of motion, one has Eq. (43).

$$
M \ddot{q}+G+\frac{\partial V}{\partial \underline{q}}=B \underline{u}+A^{T} \underline{\lambda}
$$

The inertia matrix, G matrix, B matrix, and constraints matrix can be found from the results of the previous section. If potential energy is also known, the only remaining unknowns in Eq. (43) are the generalized accelerations, the actuator torques, and the Lagrange multipliers. By using the equations of motion and the Pfaffian form of the constraints, one can eliminate the Lagrange multipliers. The time derivative of Eq. (21) is

$$
A \ddot{q}+\dot{A} \dot{q}=\mathbf{0}
$$

Solving Eq. (43) for $\ddot{q}$ and substituting the result into Eq. (44) permits one to find an expression for the Lagrange multipliers.

$$
\underline{\lambda}=\left(A M-{ }^{1} \mathrm{~A}^{\mathrm{T}}\right)^{-1}\left(A M^{-1}(G-B u)-\dot{A} \dot{q}\right)
$$

Eq. (45) can be substituted back into the equations of motion (Eq. (43)) leaving the generalized accelerations and the actuator torques as the only unknowns. As discussed in the next section, torques are found by means of inverse kinematics. Once the torques are known, the equations of motion can be integrated to find the generalized coordinates as functions of time.

\section{Inverse Kinematics}

If the motion of the system is to follow a prescribed trajectory, then the generalized accelerations at any point on that reference trajectory are known. Using reference trajectory displacements, velocities and accelerations in the reference trajectory equivalent of the equations of motion and of the Lagrange multipliers allow one to solve for the actuator torques needed to produce the reference accelerations as follows

$$
\begin{gathered}
M_{r e f-\underline{q}_{r e f}}+G_{r e f}+\frac{\partial V}{\partial \underline{q}_{r e f}}=B \underline{-}_{r e f}+A_{r e f}^{T} \lambda_{r e f} \\
\underline{\lambda}_{r e f}=\left(A_{r e f} M_{r e f}^{-1} A_{r e f}^{T}\right)^{-1}\left(A_{r e f} M_{r e f}^{-1}\left(G_{r e f}-B \underline{u}_{r e f}\right)\right. \\
\left.-A_{r e f q_{r e f}}\right)
\end{gathered}
$$

After substituting Eq. (47) into Eq. (46), the terms can be rearranged to produce equations of motion in the form

$$
\tilde{\bar{M}} \underline{\underline{q}}_{r e f}+\tilde{G}+\frac{a v}{\partial \underline{q}_{r e f}}=\bar{B}_{\underline{u}_{r e f}}
$$

where

$$
\begin{gathered}
\tilde{M}=M_{r e f} \\
\tilde{G}=G_{r e f}-A_{r e f}^{T}\left(A_{r e f} M_{r e f}^{-1} A_{r e f}^{T}\right)^{-1}\left(A_{r e f} M_{r e f}^{-1} G_{r e f}-\dot{A}_{r e f} \dot{q}_{r e f}^{(49)}\right)
\end{gathered}
$$




$$
\tilde{B}=\left(I-A_{r e f}^{T}\left(A_{r e f} M_{r e f}^{-1} A_{r e f}^{T}\right)^{-1} A_{r e f} M_{r e f}^{-1}\right) B
$$

In this study, the total number of actuators is more than the system degrees of freedom. This situation is caused by the geometric constraints of multiple manipulators handling a common object producing an excess of actuators as compared to degrees of freedom. As a result, there are an infinity of solutions for the reference torques. One method to select a specific solution is to establish a cost function. An obvious cost function is to minimize a weighted norm of the actuator torques.

$$
J=\frac{1}{2} \underline{u}_{r e f}^{T} \mathrm{~W}_{u_{-r e f}^{\mathrm{U}}}
$$

The problem now becomes one of minimizing the cost function (Eq. (52)) subject to the constraint that the reference equations of motion are satisfied (Eq. (48)). Augmenting the cost function with the constraint by means of another Lagrange multiplier leads to

$$
J=\frac{1}{2} u_{r e f}^{T} W_{u_{-r e f}^{u}}+\gamma^{T}\left(\begin{array}{l}
\tilde{B}_{-r e f}-\tilde{M} \ddot{q}_{r e f}-\tilde{G}-\frac{\partial V}{\partial V} \\
\frac{\partial q}{\partial \underline{q}_{r e f}}
\end{array}\right)
$$

The minimum of the augmented cost function is found by taking the gradient of Eq. (53) with respect to the reference torques and with respect to the Lagrange multiplier. Each of the gradients is set to zero as follows

$$
\begin{gathered}
\nabla_{\underline{u}_{r e f}} J=O=W_{u_{-r e f}^{u}}+\tilde{B}^{T} \gamma \\
\nabla_{\gamma} J=0=B \underline{u}_{r e f}-\tilde{M}_{\underline{q}}{ }_{r e f}-G-\frac{\mathrm{aV}}{\partial \underline{q}_{r e f}}
\end{gathered}
$$

Equations (54-55) are two equations in two unknowns $\left(\mathrm{y}, \underline{u}_{r e f}\right)$. Eliminating $\gamma$ results in an expression for the reference actuator torques.

$$
\underline{u}_{r e f}=W_{u}^{-1} \tilde{B}^{T}\left(\tilde{B} W_{u}^{-1} \tilde{B}^{T}\right)^{-1}\left(\tilde{M} \ddot{q}_{r e f}+\tilde{G}+\frac{u r}{\partial \underline{q}_{r e f}}\right)
$$

These values for reference actuator torques minimize the augmented cost function (Eq. (53)) at each instant in time.

\section{Lvapunoy Controller}

The equations of motion of the manipulator system are

$$
M \ddot{q}+G+\frac{\partial V}{\partial \underline{q}}=B \underline{u}+A^{T} \underline{\lambda}
$$

The constraints matrix satisfies

$$
A \underline{\dot{q}}+A_{0}=0
$$

Differentiating Eq. (58) gives

$$
\dot{A} \dot{q}+A \ddot{q}=0
$$

Solving Eq. (57)for $\ddot{q}$ results in

$$
\ddot{q}=M^{-1}\left(B u+A^{T} \underline{\lambda}-G\right)
$$

Substituting Eq. (60) into Eq. (59) produces

$$
\dot{A} \dot{q}+A M^{-1}\left(B u+A^{T} \underline{\lambda}-G\right)=0
$$

Now solve Eq. (61) for $\underline{\lambda}$

$$
\underline{\lambda}=-\left(A M-A^{T}\right)^{-1}\left\{\dot{A} \dot{q}+A M^{-1}(B \mu-G)\right\}
$$

Substitute Eq. (62) back into Eq. (60) and group terms according to the form

where

$$
q=C_{1} \underline{u}+C_{2} \dot{q}+C_{3}
$$

$$
\begin{gathered}
C_{1}=M^{-1}\left\{I-A^{T}\left(A M^{-1} A^{T}\right)^{-1} A M^{-1}\right\} B \\
C_{2}=-M^{-1} A^{T}\left(A M^{-1} A^{T}\right)^{-1} A \\
C_{3}=M^{-1}\left\{A^{T}\left(A M^{-1} A^{T}\right)^{-1} A M^{-1}-I\right\} G
\end{gathered}
$$

Similarly, the reference maneuver accelerations can be expressed as

$$
\underline{q}_{r e f}=C_{1_{r e f}-u_{r e f}}+C_{2_{r e f}} \dot{g}_{r e f} C_{3_{r e f}}
$$

where the "ref' subscripts on the $\boldsymbol{C}$ matrices indicate that reference maneuver values need to be used in their calculation. Let error quantities between the actual variables and their reference maneuver counterparts be defined by

$$
\begin{aligned}
& \delta \underline{q}=\underline{q}-\underline{q}_{r e f} \\
& \delta \underline{\dot{q}}=\underline{q}-\dot{q}_{r e f} \\
& \delta \ddot{q}=\ddot{q}-\ddot{q}_{r e f}
\end{aligned}
$$

Now define an error Lyapunov function as

$$
U=0.5(\delta \dot{q} \cdot \delta \dot{q})+f(\delta q)
$$

where $f(\delta q) \geq 0$. Differentiating Eq. (71) results in

$$
\dot{U}=\delta \underline{q} \cdot \delta \underline{q}+\sum_{i} \frac{\partial f}{\partial\left(\delta q_{i}\right)} \delta \dot{q}_{i}
$$

Let

$$
\underline{F}=\left[\frac{\partial f}{\partial\left(\delta q_{1}\right)} \frac{\partial f}{\partial\left(\delta q_{2}\right)} \cdots \frac{\partial f}{\partial\left(\delta q_{7}\right)}\right]^{T}
$$

Then Eq. (72)can be rewritten as

$$
\dot{U}=\delta \dot{q} \cdot(\delta \ddot{q}+F)
$$

Substituting Eq. (63) and Eq. (67) into Eq. (70) and then Eq. (70) into Eq. (74) produces

$$
\begin{aligned}
& \dot{U}=\delta \dot{q} \cdot\left[\left(C_{1} \underline{u}-C_{1 r e f-r e f}\right)_{+}+\left(C_{2} \underline{q}-C_{2} \dot{q}_{r e f}{ }_{r e f}\right)\right. \\
& +\left(C_{3}-C_{3 r e f}\right)+\underline{F} 1
\end{aligned}
$$


If one lets the quantity inside the brackets of Eq. (75) equal $-K_{v} \delta_{\theta}$ where $K_{v}$ is a positive definite matrix, then

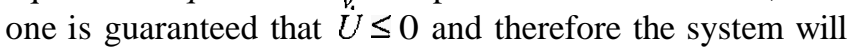
be stable in the Lyapunov sense. $K_{v}$ is assumed to be a diagonal matrix with generalized coordinate vector velocity gains on the main diagonal and zeros elsewhere. Solving Eq. (75) for command torques, $u$, leads to $\dagger$

$$
\begin{aligned}
\underline{u} & =C_{1}^{\dagger}\left[-K_{v} \delta \dot{q}+C_{1_{\text {ref ref }}}^{u}-\left(C_{2} \underline{\dot{q}}-C_{2 r e f-\dot{q}_{r e f}}\right)\right. \\
& -\left(C_{3}-C_{3 r e f}\right)-\underline{F} 1
\end{aligned}
$$

Equation (76) finds the torques that should be used rather than the reference torques. $C_{1}$ is an $8 \times 7$ matrix so $C_{1}^{\dagger}$ is its pseudo inverse. All that remains is to choose a function for $f(\delta q)$. One can chose

$$
f(\delta \underline{q})=\frac{1}{2} \delta \underline{q}^{T} K_{P} \delta \underline{q}
$$

$K_{p}$ has the same diagonal form as $K_{v}$.

\section{Reference Traiectories}

The reference trajectories describe the nominal path that the system follows in moving from the initial conditions to the desired final conditions. One need only specify reference trajectories for as many generalized coordinates as there are degrees of freedom. The choice of which generalized coordinates to specify is entirely arbitrary. A reasonable choice is any set which includes the payload coordinates and centerbody attitude since the user will probably be especially interested in these coordinates. Any path which connects the associated endpoints can be a reference trajectory. Recall, however, that the usefulness of the reference trajectories is to permit calculation of the generalized coordinates positions, velocities, and accelerations for use in the inverse kinematics calculations. To help ensure that the payload does not experience any unnecessary jerk, one might further constrain the path such that the velocities and accelerations are zero at the endpoints. Therefore, a convenient form for the reference trajectory is as a polynomial function of time. The user decides the maneuver duration in advance. The minimum order polynomial which satisfies the above boundary conditions is

$$
f(\tau)=6 \tau^{5}-15 \tau^{4}+10 \tau^{3}
$$

where $\tau$ is the normalized time.

$$
\tau=\frac{\left(t-t_{0}\right)}{\left(t_{f}-t_{0}\right)}
$$

Maneuver start and stop times are $t_{0}$ and tfrespectively.

The following equations illustrate how this fifth order polynomial reference trajectory would apply to the payload attitude generalized coordinate.

$$
\Delta \theta_{p}=\theta_{P}\left(t_{f}\right)-\theta_{P}\left(t_{0}\right)
$$

$$
\begin{gathered}
\theta_{P_{r e f}}(t)=\theta_{P}\left(t_{0}\right)+\left(6 \tau^{5}-15 \tau^{4}+10 \tau^{3}\right)\left(\Delta \theta_{P}\right) \\
\dot{\theta}_{P_{r e f}}(t)=\left(30 \tau^{4}-60 \tau^{3}+30 \tau^{2}\right)\left(\Delta \theta_{P}\right)\left(\frac{1}{t_{f}-t_{0}}\right) \\
\ddot{\theta}_{P_{r e f}}(t)=\left(120 \tau^{3}-180 \tau^{2}+60 \tau\right)\left(\Delta \theta_{P}\right)\left(\frac{1}{\left(t_{f}-t_{0}\right)^{2}}\right)
\end{gathered}
$$

Higher order polynomials can increase the complexity of the path but offer the advantage that an infinity of polynomial coefficients satisfy the position, velocity, and acceleration boundary conditions. This affords an opportunity to select the coefficients based on another optimization function. Since a reaction wheel on the centerbody will be required to maintain spacecraft attitude, the reaction wheel torque history is a prime candidate for optimization. Possible cost functions include the integral of the absolute value of reaction wheel torque or the maximum reaction wheel torque.

$$
\begin{gathered}
J=\int_{t_{o}}^{t_{f}}\left|u_{\text {wheel }}\right| d t \\
\text { or } \\
J=\max \left(\left|u_{\text {wheel }}\right|\right)
\end{gathered}
$$

\section{Results}

The system used to generate these results is a dual 2-link manipulator configuration. This system is restricted to 2-D motion in the horizontal plane to eliminate the potential energy term in Eq. (4). The system properties used for the simulations are listed in Table 1.

Four cases are presented to illustrate the system dynamics and the effect of using a reference trajectory. In all cases, the initial conditions and desired final conditions of the payload are the same. The stick figure representation of Figure 2 depicts the initial and final conditions of the desired maneuver. The triangle in the lower portion of the diagram represents the spacecraft centerbody. The five linked object with O's at the joints represents the initial manipulators and payload geometry. The object with the X's at the joint represents the desired final geometry. The desired maneuver is one in which the payload will rotate 90 degrees and its right endpoint will finish where the left endpoint started. All seven actuators are weighted evenly in the torque calculations (Eqs. (52-56))

In the first simulation, the repositioning is done entirely by the Lyapunov controller without the benefit of a reference trajectory. The behavior is that of a point controller 


\begin{tabular}{|c|c|c|}
\hline & Parameter & Value \\
\hline \multirow{7}{*}{$\begin{array}{l}\text { Length } \\
\text { (m) }\end{array}$} & $l_{L 0}$ & 0.75 \\
\hline & $l_{L 1}$ & 0.5 \\
\hline & $l_{L 2}$ & 0.5 \\
\hline & $l_{R 0}$ & 0.5 \\
\hline & $l_{R 1}$ & 0.5 \\
\hline & $l_{R 2}$ & 0.5 \\
\hline & $l_{p}$ & $0.75 \sqrt{2}$ \\
\hline \multirow{6}{*}{$\begin{array}{c}\text { Mass } \\
(\mathrm{kg})\end{array}$} & $m_{0}$ & 5 \\
\hline & $m_{L 1}$ & 1 \\
\hline & $m_{L 2}$ & 1 \\
\hline & $m_{R 1}$ & 1 \\
\hline & $m_{R 2}$ & 1 \\
\hline & $m_{P}$ & 1 \\
\hline \multirow{6}{*}{$\begin{array}{c}\text { Center } \\
\text { of } \\
\text { Mass } \\
\text { (m) }\end{array}$} & $l c_{0}$ & 0 \\
\hline & $l c_{L 1}$ & 0.25 \\
\hline & ${ }^{l} c_{L 2}$ & 0.25 \\
\hline & $l c_{R 1}$ & 0.25 \\
\hline & $l c_{R 2}$ & 0.25 \\
\hline & $l c_{p}$ & 0.25 \\
\hline \multirow{6}{*}{$\begin{array}{c}\text { Moments } \\
\text { of } \\
\text { Inertia } \\
\left(\mathrm{kg}-\mathrm{m}^{2}\right)\end{array}$} & $I_{0}$ & 5 \\
\hline & $I_{L 1}$ & 0.02083 \\
\hline & $I_{L 2}$ & 0.02083 \\
\hline & $I_{R 1}$ & 0.02083 \\
\hline & $I_{R 2}$ & 0.02083 \\
\hline & $I_{P}$ & 0.02083 \\
\hline \multirow{2}{*}{$\begin{array}{l}\text { Shoulder } \\
\text { Location } \\
\text { (deg) }\end{array}$} & $\theta_{L 0}$ & 90 \\
\hline & $\theta_{R 0}$ & 45 \\
\hline
\end{tabular}

with an initial displacement rather than that of a tracking controller. Figure 3 presents the angular displacement history. The asterisks on the right side of the plot indicate the desired final angles, Although the system is approaching the desired final geometry, it has not completely settled down even after $40 \mathrm{sec}$. Position errors are still present as well as nonzero velocities (Figure 4). Also, the reaction wheel torque is quite high during the maneuver (Figure 5). The oscillatory nature of the system is evident in the angular position and velocity plots. This behavior is also evident in Figure 6 which depicts the system geometry at several instances during the maneuver. This controller also does a poor job of maintaining the centerbody attitude. This is clearly evident in Figures $\mathbf{3}$ and 6 . The attitude error peaks at about $16 \mathrm{deg}$.

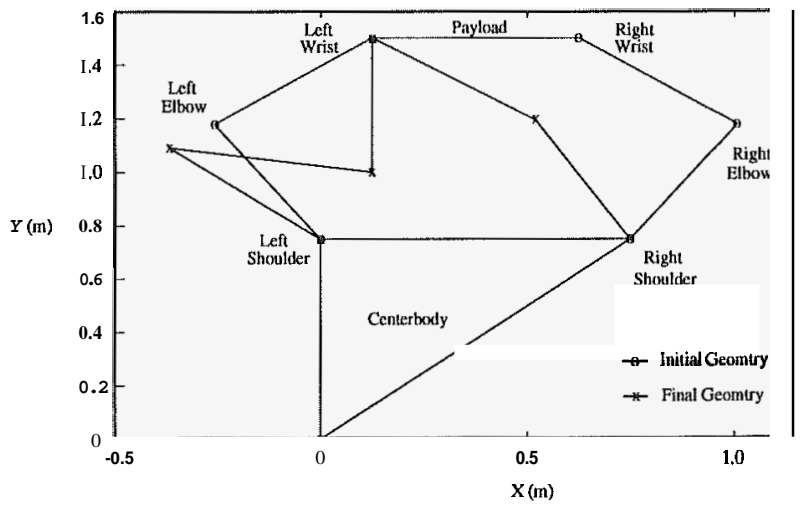

FIGURE 2. Desired Repositioning Maneuver

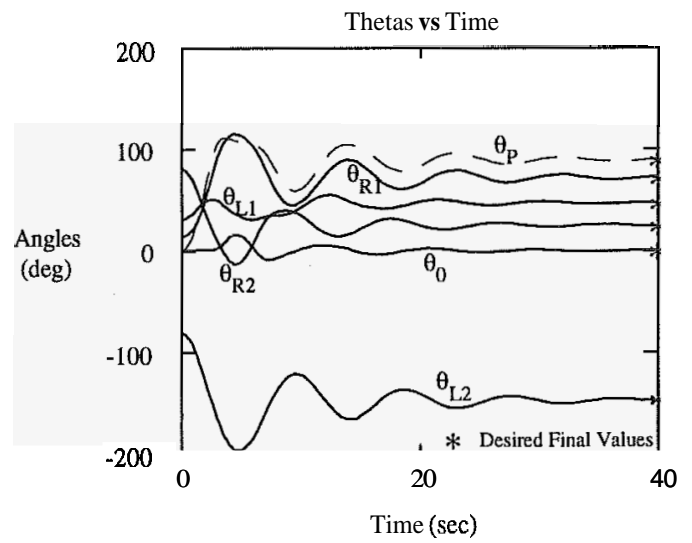

FIGURE 3. Lyapunov Point Controller Angles 


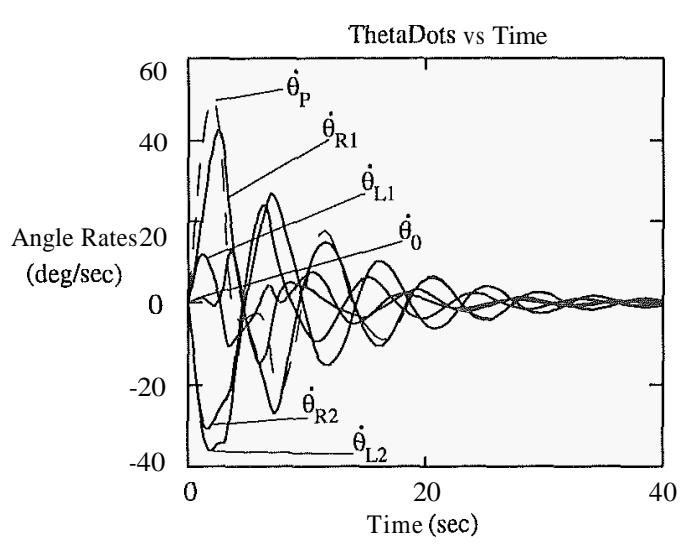

FIGURE 4. Lyapunov Point Controller Angular Rates

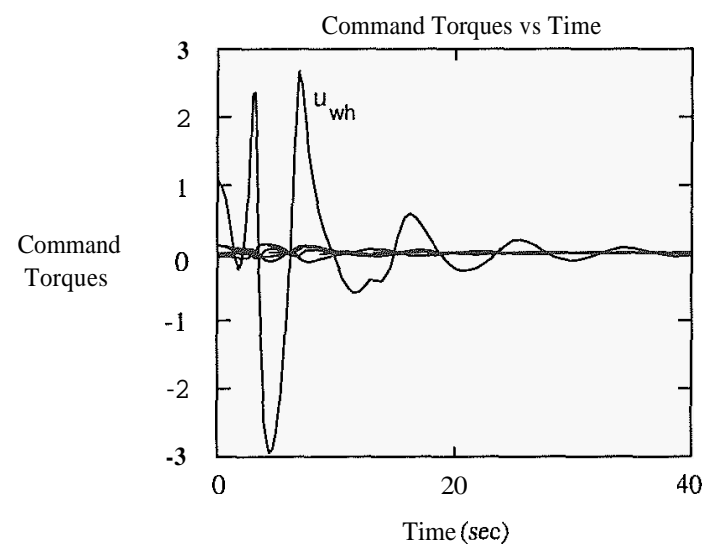

FIGURE 5. Lyapunov Point Controller Command Torques

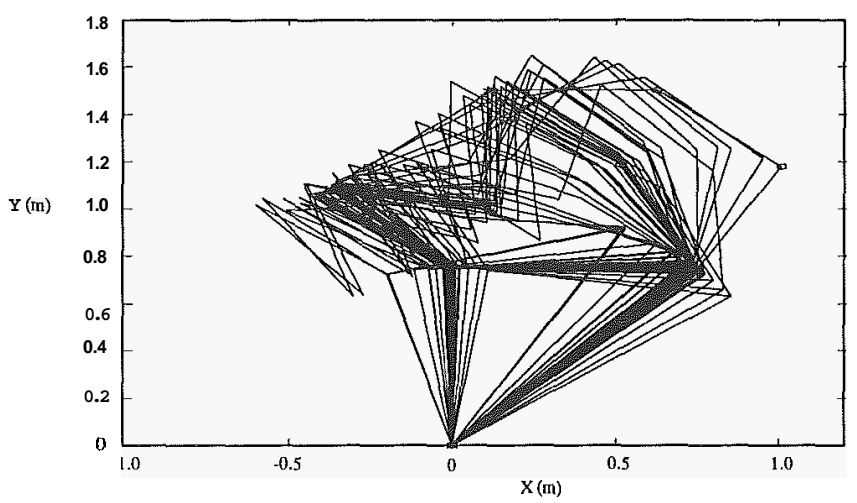

FIGURE 6. Lyapunov Point Controller Time Lapse Stick Figure

The second simulation uses a fifth order polynomial reference trajectory (Eq. (78)) applied to the payload generalized coordinates. The payload coordinates displacements, velocities, and accelerations are depicted in Figure 7. The maneuver time was selected to be $10 \mathrm{sec}$. As is evident in Figures 8 and 9 , the system successfully moves from initial conditions to desired final conditions. The command torques (Figure 10) are an order of magnitude smaller than in the previous case. More importantly, the centerbody attitude is maintained throughout the maneuver. Figure 11 shows the time lapse depiction of the maneuver.

The third simulation uses an eighth order reference trajectory polynomial and the same maneuver duration of 10 sec. The polynomial is

$$
\begin{gathered}
f(\tau)=0.0794 \tau^{8}+0.6410 \tau^{7}+0.0278 \tau^{6}+1.2764 \tau^{5} \\
-8.5973 \tau^{4}+7.5727 \tau^{3}
\end{gathered}
$$

The trajectories that result from this polynomial are shown in Figure 12. They lack the symmetry that the fifth order polynomial provided. Figures 13 through 16 indicate that the performance is still good.
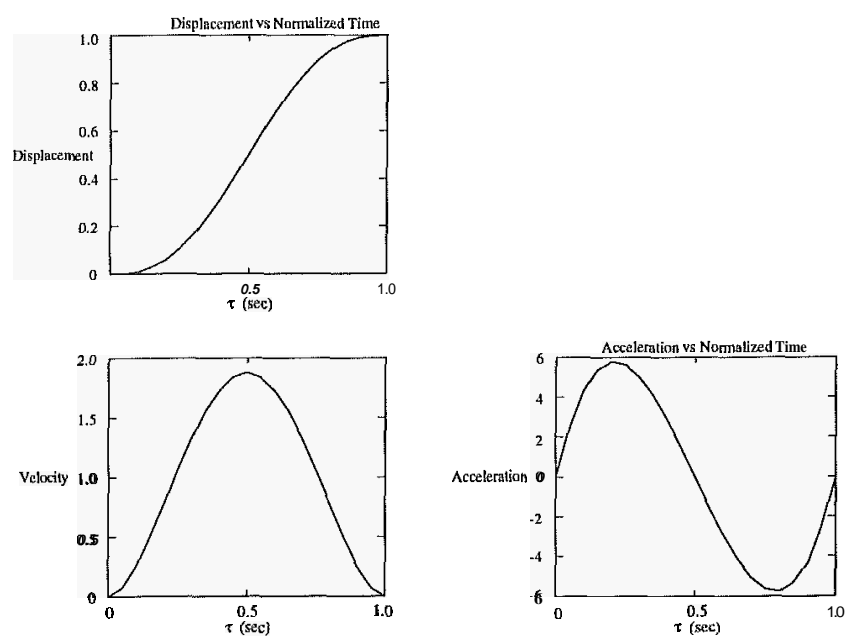

FIGURE 7. 5th Order Reference Trajectories

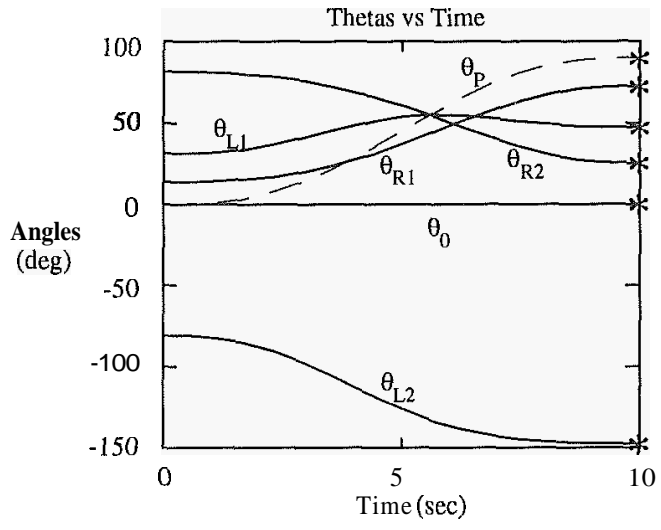

FIGURE 8. 5th Order Angles 


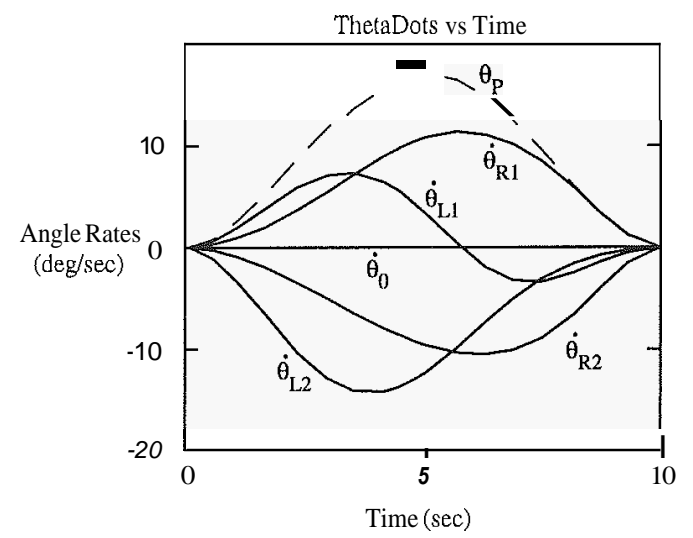

FIGURE 9. 5th Order Angular Rates

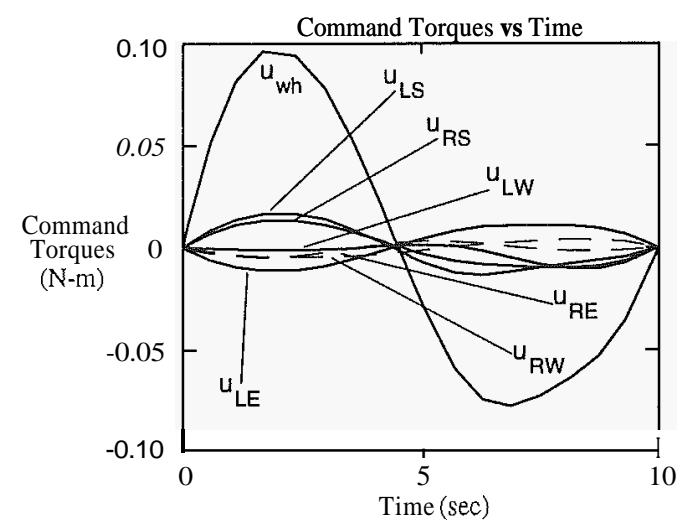

FIGURE 10. 5th Order Command Torques

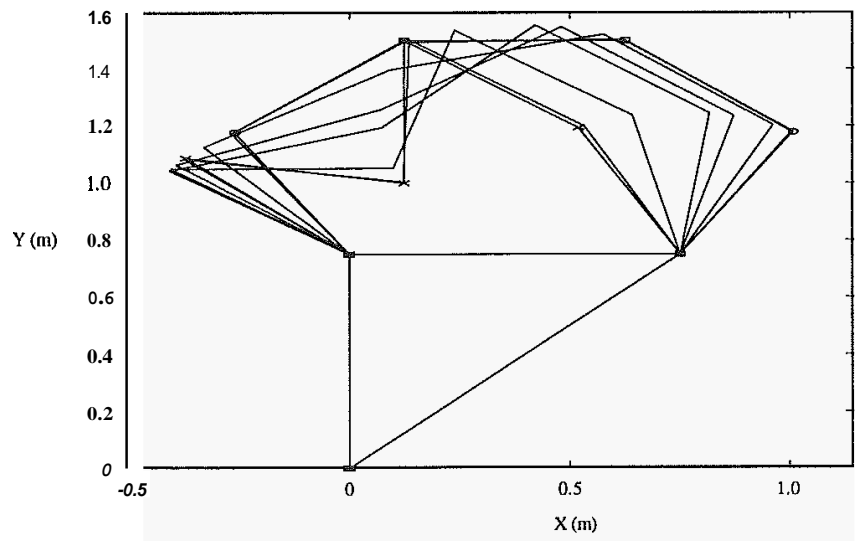

FIGURE 11. 5th Order Time Lapse Stick Figure
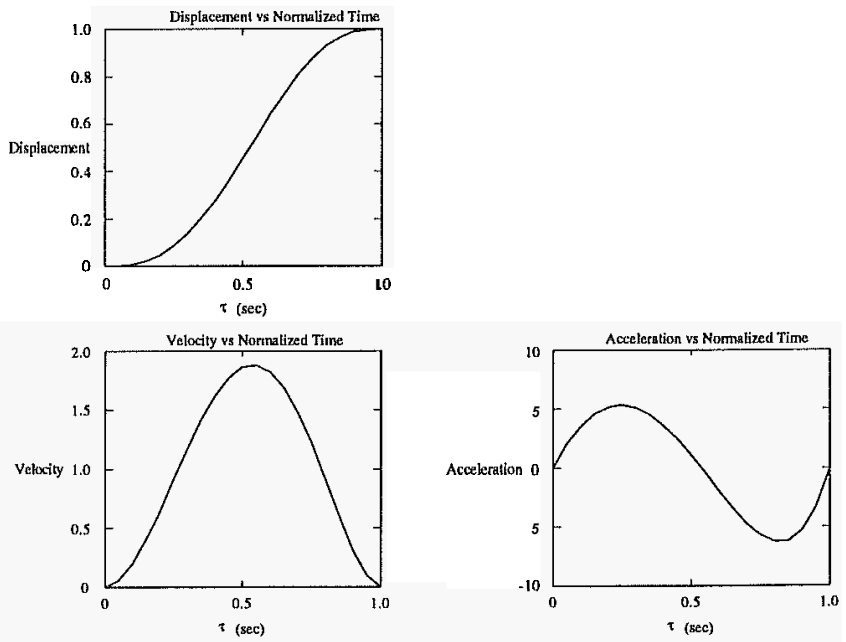

FIGURE 12. 8th Order Reference Trajectories

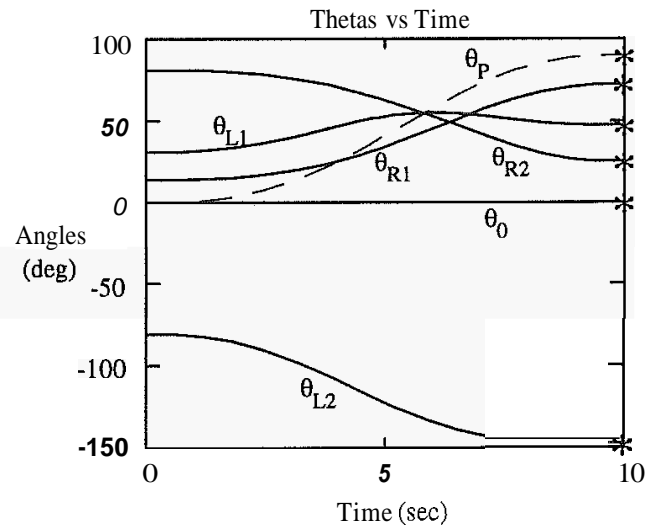

FIGURE 13. 8th Order Angles

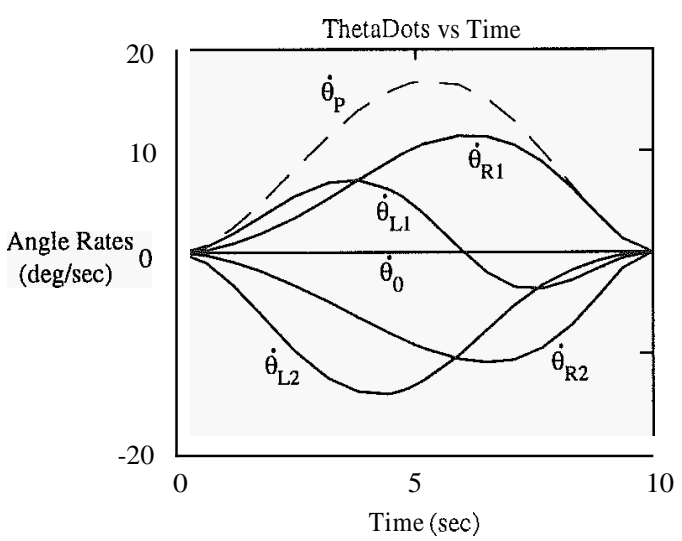

FIGURE 14. 8th Order Angular Rates 


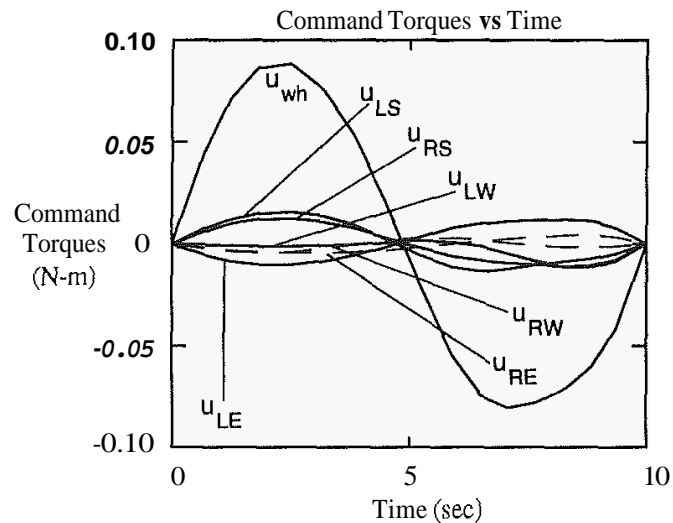

FIGURE 15. 8th Order Command Torques

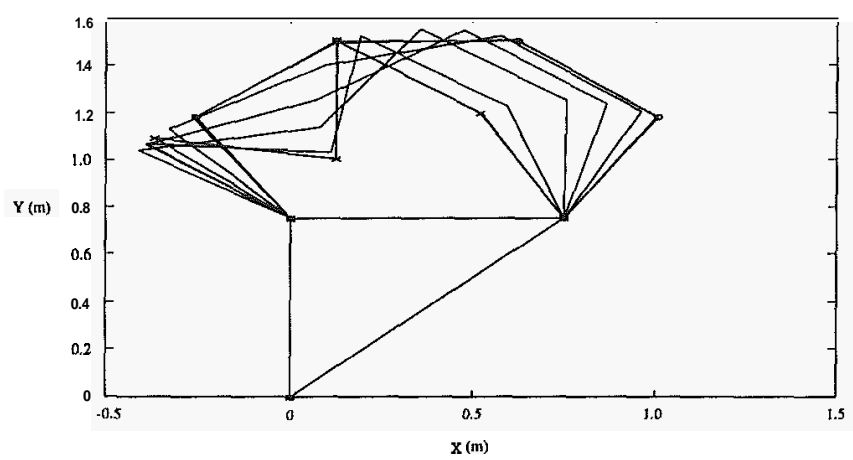

FIGURE 16. 8th Order Time Lapse Stick Figure

The fourth simulation is a modified Lyapunov controller and represents a compromise between the Lyapunov point controller and the two reference trajectory simulations. The command torques are calculated by zeroing the reference torque terms and using Eq. (76). The remaining reference trajectory terms are found using the fifth order reference trajectory. This case represents a controller analogous to a PD controller in that position and velocity errors are used to calculate the command torques. However, the gains are not constant because they are calculated from time varying matrices. As Figures 17 through 20 indicate, the performance of the modified Lyapunov controller is better than the Lyapunov point controller. The command torques are an order of magnitude smaller. This is directly attributable to using intermediate reference points on the way to a desired final state rather than attempting to achieve the desired final state all at once. Despite this dramatic improvement, performance is not as good as the reference trajectory simulations. The modified Lyapunov controller simulation takes longer to settle into the desired final state and produces an unwanted centerbody rotation (see Figure 20) of 1.2 degrees. On the other hand, the modified Lyapunov controller maintains stability.

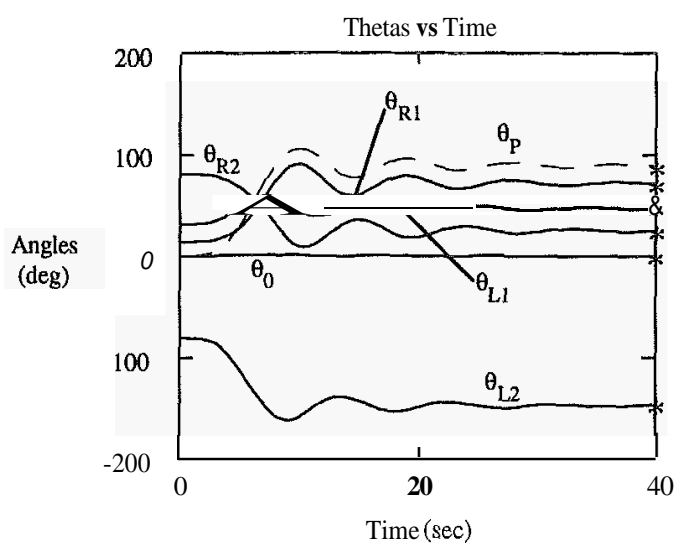

FIGURE 17. Modified Lyapunov Controller Angles

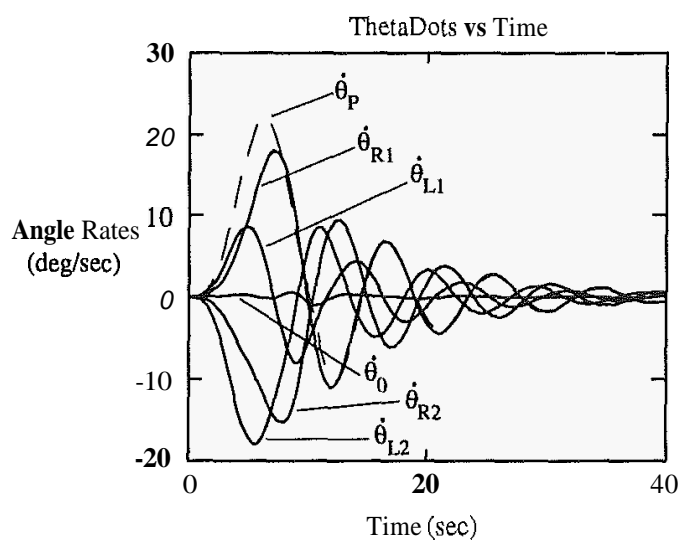

FIGURE 18. Modified Lyapunov Controller Angular Rates

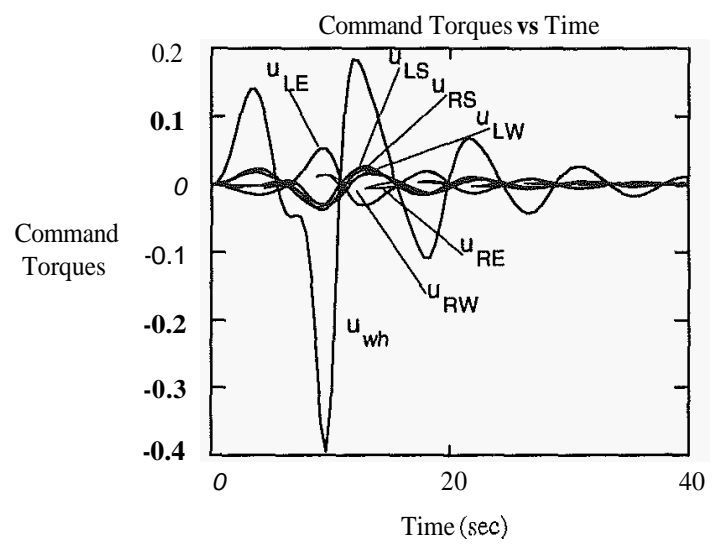

FIGURE 19. Modified Lyapunov Controller Command Torques 


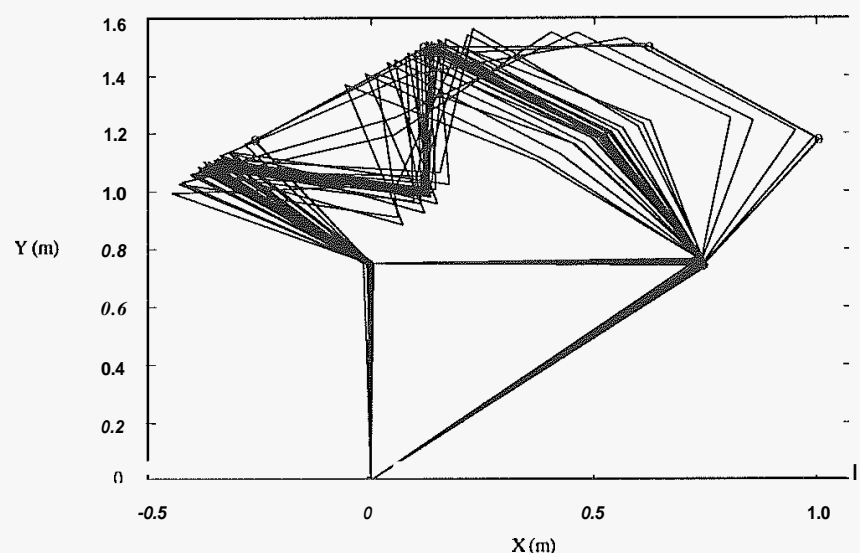

FIGURE 20. Modified Lyapunov Controller Time Lapse Stick Figure

Comparing the values produced by integrating the absolute value of the reaction wheel torque (Eq. (84)) for the four simulations provides a means to distinguish between the cases. A second metric is the absolute value of the maximum reaction wheel torque (Eqn 85). Another obvious choice is to bound the centerbody attitude error during each simulation. The results are summarized in Table 2. Clearly the point controller is the worst controller based on all three metrics. The difference between the fifth and eighth order tracking controllers is only slight.

\begin{tabular}{|c|c|c|c|c|}
\hline & & $\int\left|u_{w h}\right| d t$ & $\left|u_{\max }\right|$ & $\begin{array}{l}\text { Centerbody } \\
\text { Attitude } \\
\text { Error (deg) }\end{array}$ \\
\hline \multicolumn{2}{|c|}{ Point Controller } & 17.3841 & 2.9365 & 16.2261 \\
\hline \multirow{2}{*}{$\begin{array}{l}\text { Tracking } \\
\text { Controller }\end{array}$} & $5^{\text {th }}$ Order & 0.5746 & 0.0961 & 0.0000 \\
\hline & $8^{\text {th }}$ Order & 0.5705 & 0.0885 & 0.0000 \\
\hline \multicolumn{2}{|c|}{$\begin{array}{l}\text { Modified Tracking } \\
\text { Controller }\end{array}$} & 2.4523 & 0.3950 & 1.1910 \\
\hline
\end{tabular}

TABLE 2. Comparisons of Simulations

\section{Conclusions}

This paper addresses the problem of repositioning a payload that is grasped by multiple manipulators mounted on the same free floating base. The closed chain nature of the problem allows for an infinite set of joint actuator torques to accomplish the maneuver. This paper presented a technique whereby a weighted norm approach selects a torque profile to use. Use of polynomial reference trajectory significantly improves the performance of the system. As the order of the polynomial increases, the redundancy of the coefficients can be used to select values that lead to reduced centerbody attitude disturbance. The biggest improvement is from including the polynomial trajectory in the first place. The minimal improvement achieved by increasing the order of the polynomial probably doesn't warrant the additional computational expense.

\section{References}

[1] Robert E. Lindberg, Richard W. Longman, and Michael F. Zedd, "Kinematic and Dynamic Properties of an Elbow Manipulator Mounted on a Satellite," The Journal of the Astronautical Sciences, Vol. 38, No. 4, October-December 1990, pp. 397-421.

[2] Harold L. Alexander and Robert H. Cannon Jr., "An Extended Operational-Space Control Algorithm for Satellite Manipulators," The Journal of the Astronautical Sciences, Vol. 38, No. 4, October-December 1990, pp. 473-486.

[3] Richard W. Longman, Robert E. Lindberg, and Michael F. Zedd, "Satellite-Mounted Robot Manipulators -- New Kinematics and Reaction Moment Compensation," The International Journal of Robotics Research, Vol. 6, No. 3, pp. 87-103, Fall 1987.

[4] C. L. Chung, S. Desa, and C. W. deSilva, "Base Reaction Optimization of Redundant Manipulators for Space Applications," Report No. NASA-CR-186274, 1988.

[5] Z. Vafa, "Space Manipulator Motions with No Satellite Attitude Disturbances," Proceedings of 1990 IEEE International Conference on Robotics and Automation, Cincinnati, Ohio, 1990, pp. 1770-1775.

[6] K. Yoshida, R. Kurazume, and Y. Umetani, "Torque Optimization Control in Space Robots with a Redundant Arm," Proceedings of IROS '91, Osaka, Japan, 1991, pp. 1647-1652.

[7] Shaheen Ahmad and Mohamed Zribi, "Lyapunov Based Control Design for Multiple Robots Handling a Common Object," Mechanics and Control: Proceedings of 4th Workshop on Mechanics and Control, University of Southern California, Los Angeles, Jan. 2123, 1991, edited by J. M. Skowronski, H. Flashner, and R. S. Guttaul, pp. 1-17, Springer-Verlag, 1992.

[8] Stanley A. Schneider and Robert H. Cannon Jr., "Object Impedance Control for Cooperative Manipulation: Theory and Experimental Results," Proceedings of 1989 IEEE International Conference on Robotics and Automation, Scotsdale, Arizona, 1989, pp. 10761083. 